\title{
Using the Euler-Maruyama Method for Finding a Solution to Stochastic Financial Problems
}

\author{
Hamid Reza Erfanian \\ Department of Mathematics, University of Science and Culture, Tehran, Iran. \\ E-mail: Erfanian@usc.ac.ir \\ Mahs hid Hajimohammadi and Mohammad Javad Abdi \\ Department of Mathematics, University of Science and Culture, Tehran, Iran. \\ E-mail: mahshidhajimohammadi70@gmail.com; abdi.mohammad.javad@gmail.com
}

\begin{abstract}
The purpose of this paper is to survey stochastic differential equations and Euler-Maruyama method for approximating the solution to these equations in financial problems. It is not possible to get explicit solution and analytically answer for many of stochastic differential equations, but in the case of linear stochastic differential equations it may be possible to get an explicit answer. We can approximate the solution with standard numerical methods, such as Euler-Maruyama method, Milstein method and Runge-Kutta method. We will use Euler-Maruyama method for simulation of stochastic differential equations for financial problems, such as asset pricing model, square-root asset pricing model, payoff for a European call option and estimating value of European call option and Asian option to buy the asset at the future time. We will discuss how to find the approximated solutions to stochastic differential equations for financial problems with examples.
\end{abstract}

Index Iterms - Stochastic Differential Equations, EulerMaruyama method, Asset pricing model, Square-Root as set pricing model.

\section{INTRODUCTION}

Stochastic differential equation (SDE) models play a prominent role in a range of application areas, including chemistry, biology, mechanics, economics and finance [1]. These equations have become standard models for financial quantities such as as set prices, interest rate and their derivatives [2]. Ito and Stratonovich put stochastic differential equations in mathematics. Ito pioneered the theory of stochastic integral and stochastic differential equations, it use in various filed such as financial mathematics. To understand the dynamics of most SDEs and their solutions, it is important to have some knowledge of probability theory as well as some mathematica $/$ statistical principles [3]. It is not possible to get explicit solution to many of stochastic differential equations. So, we approximate solution with numerical methods. We have based this paper around financial examples. At first, we introduce option, Brownian motion and compute discretized Brownian paths, Ito stochastic integral and stochastic differential equations. Then we survey how the Euler-Maruyama method simulate a stochastic differential equation in financial problems.

Euler method is a method for solving ordinary differential equations (ODEs) with a given initial value, it is named after Leonhard Euler who treated this method in his book (Institutionum calculi integral is published 176870). Gisiro Maruyama in 1995 showed unique answer for stochastic differential equations with Eu ler appro ximation and he proved the mean-square convergence of the Euler approximation of the Ito process without jumps, this being one of the first papers on the approximation of Ito processes [4]. Euler-Maruyama method is named after Leonhard Euler and Gisiro Maruyama.

\section{PRELIMINARIES}

In this section, we review some of the basic concepts and definitions which are used in this paper.

\section{A. Option}

An option is a contract which gives the owner or holder the right to buy (call option) or sell (put option) a specified number of shares of an underlying asset at a fixed price (strike price, denoted as $K$ ) before a specified future date or on the specified date. The time that option expires is called expiry, denoted as $T$. One of the most common types of options, is European option. A European option can only be exercised at its maturity. This option gives its owner the right, to buy one unit of a particular asset at a pre-set price ( $K$ ), at some fixed future time ( $T$ ). If the price of the asset at time $T$ is lower than $K$, the option value is zero and it will not be exercised. Otherwise, if the price of the ass et at time $T$ exceeds $K$, the owner of the option may buy one unit of the asset at price $K$ and immediately sell it at price $S(T)$, so gaining a profit of $S(T)-K$. As a result, the payoff of the European call option is:

$$
(S(T)-K)^{+}= \begin{cases}S(T)-K & \text { if } S(T)>K, \\ 0 & , \text { if } S(T) \leq K\end{cases}
$$


Hence, the payoff from purchasing a European call option can be represented by the $C=\max (S(T)-k, 0)$.

"Fig. 1," shows payoff for a European call option with strike price $\$ 30$. Another type of option is Asian option or average option. For Asian options the payoff is determined by the average price A of the underlying as set over some pre-specified period of time as opposed to at maturity. Asian call payout is:

$$
P(T)=\max (A(0, T)-K, 0),
$$

where $A$ denote the average, and $K$ the strike price. The average $A$ may be shown in many ways. For the case of discrete monitoring with monitoring at time $t_{1}, \ldots, t_{N}$, the average is:

$$
A(0, T)=\frac{1}{N} \sum_{i=1}^{N} S\left(t_{i}\right) .
$$

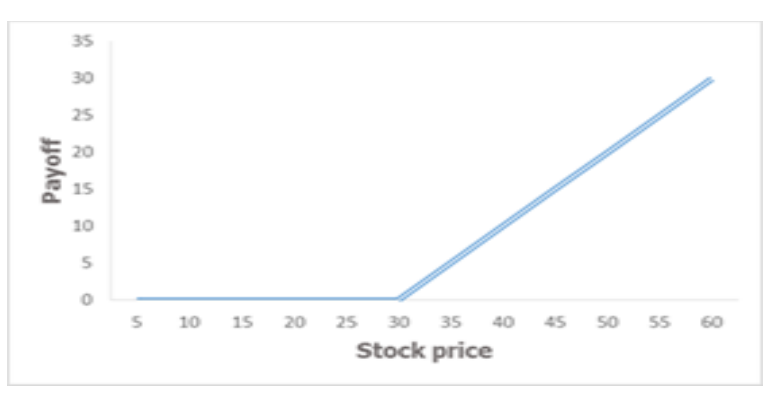

Fig.1. Payoff for European call option with strike price $\$ 30$.

\section{B. Brownian motion}

A Brownian motion, or Wiener process, over $[0, T]$ is a random variable $W(t)$ that depends continuously on $t \in[0, T]$ and satisfies the following conditions:

1. $W(0)=0$, With probability 1 .

2. For $0 \leq v \leq t \leq T$ the random variable given by the increment $W(t)-W(v)$ is normally distributed $N(0, t-v)$ and $W(t)-W(v) \sim \sqrt{t-v} N(0,1)$ where $N(0,1)$ denote normally distributed random variable with zero mean and unit variance.

3. For $0 \leq v<t<u<s \leq T$ the increments $W(t)-W(v)$ and $W(s)-W(u)$ are independent.

We consider discretized Brownian motion for computational purposes, where $W(t)$ is specified at discrete $t$ values. We set $\delta t=T / N$ (for some positive integer $N)$. $W_{j}$ Denote $W\left(t_{j}\right)$ with $t_{j}=j \delta t$. We have $W_{0}=0$ with probability 1 from condition 1 , from conditions 2 and 3 :

$$
W_{j}=W_{j-1}+d W_{j}, j=1, \ldots, N
$$

where each $d W_{j}$ is an independent random variable of the form $\sqrt{\delta t} N(0,1)$ [1]. MATLAB 1 [1] performs one simulation of discretized Brownian motion over $[0,1]$ with $N=400$, and produce "Fig. 2". Now, we simulate the stochastic function

$$
u(W(t))=e^{\left(t+\frac{1}{2} W(t)\right)}
$$

along 2000 discretized Brownian paths over [0,1] with $N=400$. Equation (5) has the form of the answer of linear stochastic differential equations. "Fig. 3," show average of $u(W(t))$ over 2000 discretized Brownian paths. The average of $u(W(t))$ is plotted with a solid green line. 5 individual paths are plotted with a dashed red line.

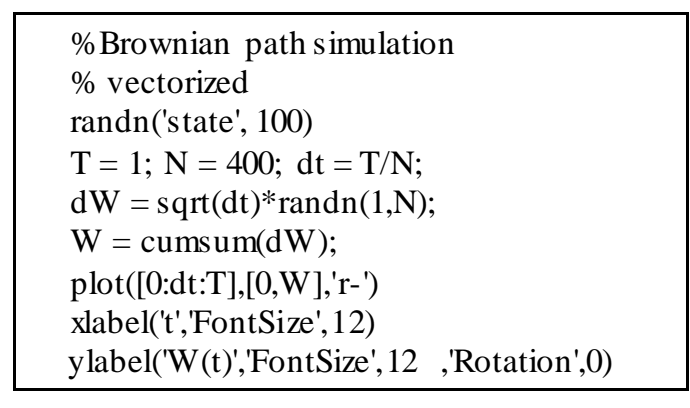

MATLAB 1. Simulation of Brownian path.

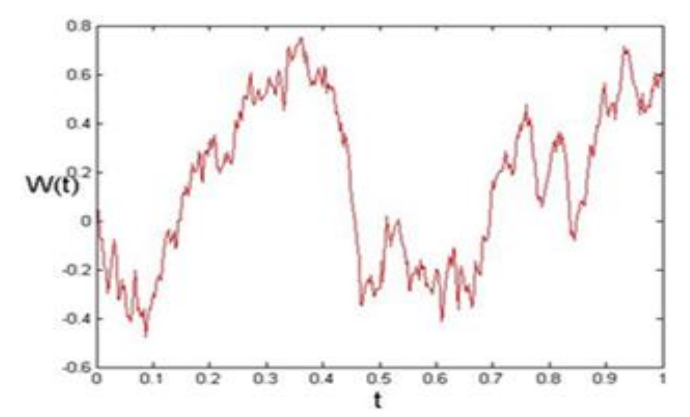

Fig. 2. Simulation of discretized Brownian path over $[0,1]$ with $\mathrm{N}=400$.

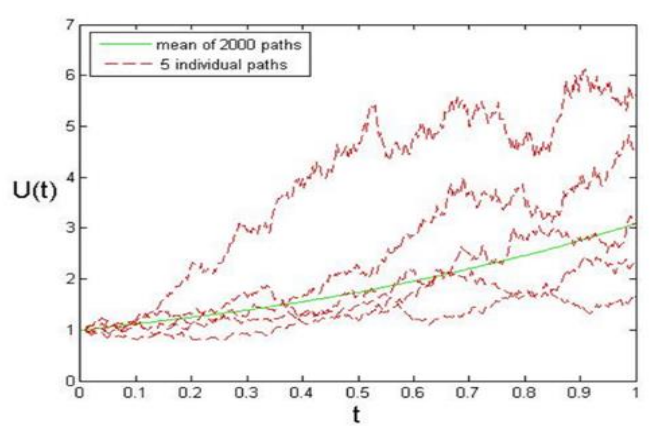

Fig.3. $u(W(t))$ averaged over 2000 discretized Brownian paths along 5 individual paths

In "Fig. 3," the maximum discrepancy between the sample average and the expected value of $u(W(t))\left(e^{\frac{9 t}{8}}\right)$ over all point $t_{j}$ is 0.0177 . 


\section{Ito integral}

Let $0=t_{0}<t_{1}<\ldots<t_{N-1}<t_{N}=T$ be a grid of points on the interval $[0, T]$, The Ito integral is the limit:

$$
\int_{0}^{T} h(t) d W(t)=\lim _{\delta t \rightarrow 0} \sum_{j=0}^{N-1} h\left(t_{j}\right)\left(W\left(t_{j+1}\right)-W\left(t_{j}\right)\right)
$$

\section{Ito formula}

If $Y=f(t, X)$, then

$$
d Y=\frac{\partial f}{\partial t}(t, X) d t+\frac{\partial f}{\partial x}(t, X)+\frac{1}{2} \frac{\partial^{2} f}{\partial x^{2}}(t, X) d x d x
$$

Where $d x d x$ term is interpreted as follows:

$$
\begin{aligned}
& d t d t=0, \\
& d t d W_{t}=d W_{t} d t=0, \\
& d W_{t} d W_{t}=0 .
\end{aligned}
$$

\section{STOCHASTIC DIFFERENTIAL EQUATIONS}

Stochastic differential equation (SDE) is a differential equation in which some of the terms and its solution are stochastic processes [5]. SDEs are important in filtering problems, stochastic approaches to deterministic boundary value problems, optimal stopping, stochastic control, and financial mathematics [6]. A general form for a stochastic differential equation is:

$$
\begin{aligned}
& d X(t)=f(X(t)) d t+g(X(t)) d W(t), \\
& X(0)=X_{0}, \\
& 0 \leq t \leq T .
\end{aligned}
$$

Where $W$ denote a Brownian motion and $f$ and $g$ are given functions. A solution is a stochastic process, which can be interpreted as integral equation [7]:

$$
\begin{aligned}
& X(t)=X_{0}+\int_{0}^{t} f(X(s)) d s+\int_{0}^{t} g(X(s)) d W(s), \\
& 0 \leq t \leq T .
\end{aligned}
$$

The second integral is Ito integral. If $g \equiv 0$ and $X_{0}$ is constant, (10) reduces to the ordinary differential equation:

$$
\frac{d X}{d t}=f(X(t)), X(0)=X_{0}
$$

To better understand the difference between (9) and (11) we suppose that a person has two possible investments:
1. A risky investment e.g. a stock, where the price $P_{1}(t)$ per unit at time $t$ satisfies a stochastic differential equation:

$$
\frac{d p_{1}}{d t}=(a+\alpha . " \text { noise" }) P_{1}
$$

Where $a>0$ and $\alpha \in \mathbb{R}$ are constants.

2. A safe investment e.g. a bond, where the price $P_{2}(t)$ per unit at time $t$ grows exponentially:

$$
\frac{d P_{2}}{d t}=b P_{2}
$$

where $b$ is a constant, $0<b<a[8]$.

Equation (12) is stochastic differential equation, we don't know the "noise" behavior. An SDE is an important model in science and engineering when noise affects behavior. For example, SDEs can model the rapidly fluctuating prices of the stock market [9]. But in (13) we don't have the "noise" and (13) is ordinary differential equation. . The well- known Black-Scholes model of the as set price is described by the linear SDE [10]:

$$
\begin{aligned}
& d X(t)=\mu X(t) d t+\sigma X(t) d W(t), \\
& X(0)=X_{0}
\end{aligned}
$$

where $\mu$ and $\sigma$ are real constants. This stochastic differential equation arises, for example, as an asset pricing model in financial mathematics. The general solution of a linear stochastic differential equation can be found exp licitly [11].The solution of the (14) is geometric Brownian motion [12]:

$$
X(t)=X_{0} \exp \left(\left(\mu-\frac{1}{2} \sigma^{2}\right) t+\sigma W(t)\right)
$$

Geometric Brownian Motion is nonnegative, it provides for a more realistic model of stock prices. Also, the Geometric Brownian Motion model considers the ratio of stock prices to have the same normal distribution. [13]. An alternative to the stock price model (14) is the mean reverting square root process [14]:

$$
d S_{t}=a\left(b-S_{t}\right) d t+c \sqrt{S_{t}} d W_{t},
$$

where $\mathrm{a}, \mathrm{b}$ and $\mathrm{c}$ are constant. To check solution (15) for (14) [2], write

$$
X=f(t, Y)=X_{0} e^{Y},
$$

where $Y=\left(\mu-\frac{1}{2} \sigma^{2}\right) t+\sigma W_{t}$. By the (9), 


$$
d X=X_{0} e^{Y} d Y+\frac{1}{2} e^{Y} d Y d Y
$$

where $d Y=\left(\mu-\frac{1}{2} \sigma^{2}\right) d t+\sigma d W_{t}$. By the (10),

$$
d Y d Y=\sigma^{2} d t
$$

As a result

$$
\begin{aligned}
d X & =X_{0} e^{Y}\left(\mu-\frac{1}{2} \sigma^{2}\right) d t+X_{0} e^{Y} \sigma d W_{t}+\frac{1}{2} \sigma^{2} X_{0} e^{Y} d t \\
& =X_{0} e^{Y} \mu d t+X_{0} e^{Y} \sigma d W_{t} \\
& =\mu X d t+\sigma X d W_{t}
\end{aligned}
$$

We use Monte Carlo simulation for linear stochastic differential equation at following example.

Example 1. Suppose following process for the stock price $S$ :

$$
d S=0.16 S d t+0.32 S d W
$$

where the stock pays no dividends, has a volatility of $32 \%$ per annum, the expected return from a stock is $16 \%$ per annum with continuous compounding and the initial stock price is $\$ 100\left(S_{0}=100\right)$. According to Wiener process, $d W$ is $\varepsilon \sqrt{\Delta t}$ where $\varepsilon \sim N(0,1)$. Let $\Delta S$ is the increase in the stock price in the next s mall interval of time. So,

$$
\Delta S=0.16 S \Delta t+0.32 S \varepsilon \sqrt{\Delta t}
$$

Consider a time interval of one week or $1 / 52$ of a year. So,

$$
\Delta S=0.00307 S+0.04434 S \varepsilon
$$

Table 1. Monte Carlo simulation of stock price where $\mu=0.16$ and $\sigma=0.32$ during 1 -week periods over fifteen weeks.

\begin{tabular}{|c|c|c|}
\hline $\begin{array}{c}\text { Stock price } \\
\text { (at start of period) }\end{array}$ & $\begin{array}{c}\text { Random sample } \\
(\varepsilon)\end{array}$ & $\begin{array}{c}\text { Change in stock price } \\
\text { during period }\end{array}$ \\
\hline 100 & -0.66 & -2.62 \\
\hline 97.38 & -0.46 & -1.69 \\
\hline 95.69 & 0.28 & 1.48 \\
\hline 97.17 & 0.27 & 1.46 \\
\hline 98.63 & 0.84 & 3.98 \\
\hline 102.61 & 0.65 & 3.27 \\
\hline 105.88 & 0.95 & 4.78 \\
\hline 110.66 & 0.52 & 2.89 \\
\hline 113.55 & 0.86 & 4.68 \\
\hline 118.23 & -0.71 & -3.36 \\
\hline 114.87 & -0.44 & -1.89 \\
\hline 112.98 & -0.24 & -0.85 \\
\hline 112.13 & 0.91 & 4.87 \\
\hline 117 & 1.58 & 8.55 \\
\hline 125.55 & 0.54 & 3.39 \\
\hline 128.94 & 0.78 & 4.85 \\
\hline
\end{tabular}

To produce a random sample ${ }_{\varepsilon}$ from a standard normal distribution in Excel, we use NORMSINV $(R A N D())$ [15]. We use Monte Carlo simulation of stock price over fifteen weeks for this example in Table 1 . So, the random sample from the distribution of stock prices at the end of $15^{\text {th }}$ week is 128.94 .

\section{EULER-MARUYAMA METHOD}

There is a stochastic analog of Euler's method which is known as the Euler-Maruyama method [6]. EulerMaruyama method is constructed within the Ito integral framework [7]. To apply Euler-Maruyama method to (9) over $[0, T]$, we first divide the interval. Let $\Delta t=T / L$ (for some positive integer $L)$ and $\tau_{j}=j \Delta t(j=0, \ldots, L)$. Our numerical approximation to $X\left(\tau_{j}\right)$ will be denoted $X_{j}$. The Euler-Maruyama method takes the form [1]:

$$
\begin{aligned}
& X_{j}=X_{j-1}+f\left(X_{j-1}\right) \Delta t+g\left(X_{j-1}\right)\left(W\left(\tau_{j}\right)-W\left(\tau_{j-1}\right)\right), \\
& j=1, \ldots, L
\end{aligned}
$$

Now we want to obtain (24). Setting $t=\tau_{j}$ and $t=\tau_{j-1}$ in (10), we obtain:

$$
\begin{gathered}
X\left(\tau_{j}\right)=X_{0}+\int_{0}^{\tau_{j}} f(X(s)) d s+\int_{0}^{\tau_{j}} g(X(s)) d W(s) \\
X\left(\tau_{j-1}\right)=X_{0}+\int_{0}^{\tau_{j-1}} f(X(s)) d s+\int_{0}^{\tau_{j-1}} g(X(s)) d W(s) .
\end{gathered}
$$

We subtract these equations ((25) and (26)):

$$
X\left(\tau_{j}\right)=X\left(\tau_{j-1}\right)+\int_{\tau_{j-1}}^{\tau_{j}} f(X(s)) d s+\int_{\tau_{j-1}}^{\tau_{j}} g(X(s)) d W(s) .
$$

For the first integral in (27) we can use the conventional deterministic quarture approximation and for second integral we use Ito formula [7]:

$$
\begin{gathered}
\int_{\tau_{j-1}}^{\tau_{j}} f(X(s)) d s \approx\left(\tau_{j}-\tau_{j-1}\right) f\left(X_{j-1}\right)=\Delta t f\left(X_{j-1}\right) . \\
\int_{\tau_{j-1}}^{\tau_{j}} g(X(s)) d W(s) \approx g\left(X_{j-1}\right)\left(W\left(\tau_{j}\right)-W\left(\tau_{j-1}\right)\right) .
\end{gathered}
$$

Setting (29) and (28) in (27) gives us Euler-Maruyama formula (24).The Euler-Maruyama method has strong order of convergence $1 / 2$ and weak order of convergence 1 [16]. Now, we use this method for approximating stochastic differential equations in financial problems.

Example 2. Suppose following process for the stock price:

$$
\begin{aligned}
& d S_{t}=0.16 S_{t} d t+0.30 S_{t} d W_{t}, \\
& S_{0}=100 .
\end{aligned}
$$


$S$ is the stock price at a particular time and $W_{t}$ is Brownian motion. The exact solution to this equation is:

$$
S_{t}=100 \exp \left(0.115 t+0.30 W_{t}\right)
$$

We have a discretized Brownian path over [0,1] with $\delta t=2^{-8}$. The solution (31) is plotted with magenta line in Fig 4. We apply Euler-Maruyama method to (30) using a step size $\Delta t=R \delta t$ with $R=32$, that is plotted as green asterisks connected with dashed lines in "Fig. 4". The discrepancy between the exact solution (31) and EulerMaruyama approximation at the endpoint $t=T=1$, was found to be 3.8741. Using smaller $\mathrm{R}$ values of $8(R=8)$, shown in "Fig. 5," (see MATLAB 2), produced endpoint errors of 0.5084 . So, the endpoint errors decreased with smaller R. For better conclusion we use values of 2 $(R=2)$, shown in "Fig. 6".

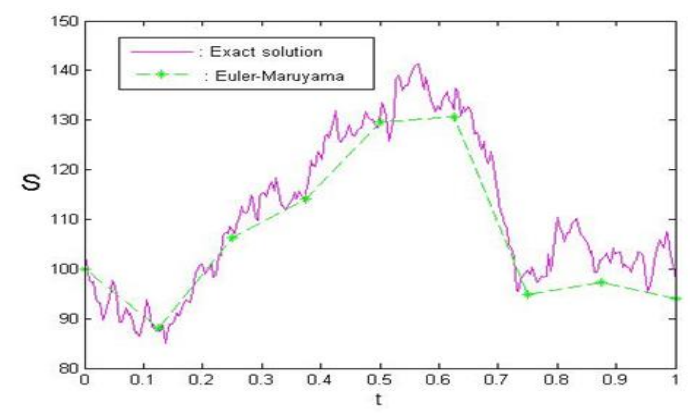

Fig.4. Exact solution and the solution based on Euler-Maruyama approximation with step size $\Delta t=R \delta t, R=32$.

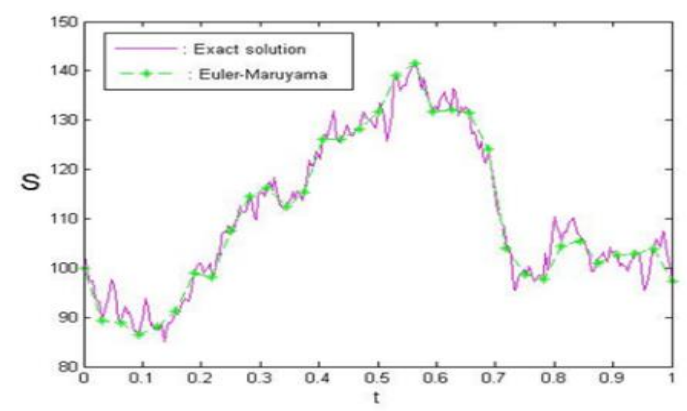

Fig.5. Exact solution and the solution based on Euler-Maruyama approximation with step size $\Delta t=R \delta t, R=8$.

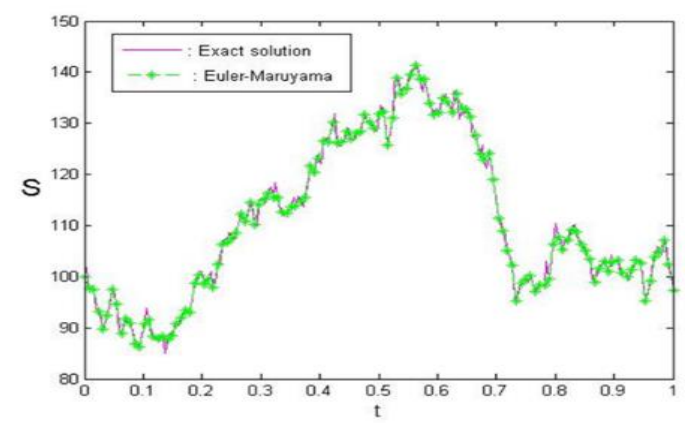

Fig.6. Exact solution and the solution based on Euler-Maruyama approximation with step size $\Delta t=R \delta t, \boldsymbol{R}=2$.
Example 3. We apply Euler-Maruyama method to a square-root asset pricing model with $\mu=0.09, \sigma=0.6$ and $S_{0}=25$ :

$$
\begin{aligned}
& d s_{t}=0.09 S_{t} d t+0.6 \sqrt{S_{t}} d W_{t} \\
& 0 \leq t \leq 1
\end{aligned}
$$

We approximate this stochastic differential equation (32), for a single path but with different step sizes $\left(2^{-4}, 2^{-5}, 2^{-6}\right.$ and $\left.2^{-7}\right)$ [14] are shown in "Fig. 7". According to the Example 1, we conclude that the approximation with the smallest $\Delta t$ is the best approximation. So, the red asterisks connected with dashed line is the best approximation.

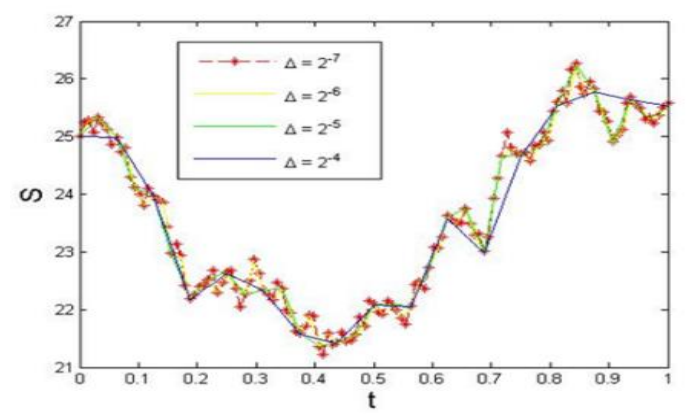

Fig.7. Euler-Maruyamaapproximation to a square-root asset pricing model with different step sizes.

\section{THE APPROXIMATION PAYOFF FOR A EUROPEAN CALL OPTION}

Let $f(t, S(t))$ represent the value of a European call option on stock $S$. Let $S$ follow the equation

$$
d S=S r d t+S \sigma d W
$$

We want to compare Eu ler-Maruyama method and true solution of the payoff at the initial time. We compute the true solution and Euler-Maruyama approximation along 2000 discretized Brownian paths for different initial values, ranging from 70 to 95 . The strike price $K$ set at 83 , the interest rate at $10.9 \%$, the time $T$ at 0.2 years and $\sigma=0.1195$ (volatility). Look at "Fig. 8", it resembles the call payoff graph shown in "Fig. 1". The black line represents the true solution and the $\mathrm{x}$-green line represents the Euler-Maruyama approximation. The graph's curve in "Fig. 8," begins to rise before the strike price ( $K=83$ ), that differs from the previously shown payoff graph in "Fig. 1". This difference can be explain by the fact that graph in "Fig. 8," is for the expected value of the payoff at the initial time, while the previously shown payoff graph was drawn at the expiration date [13]. 


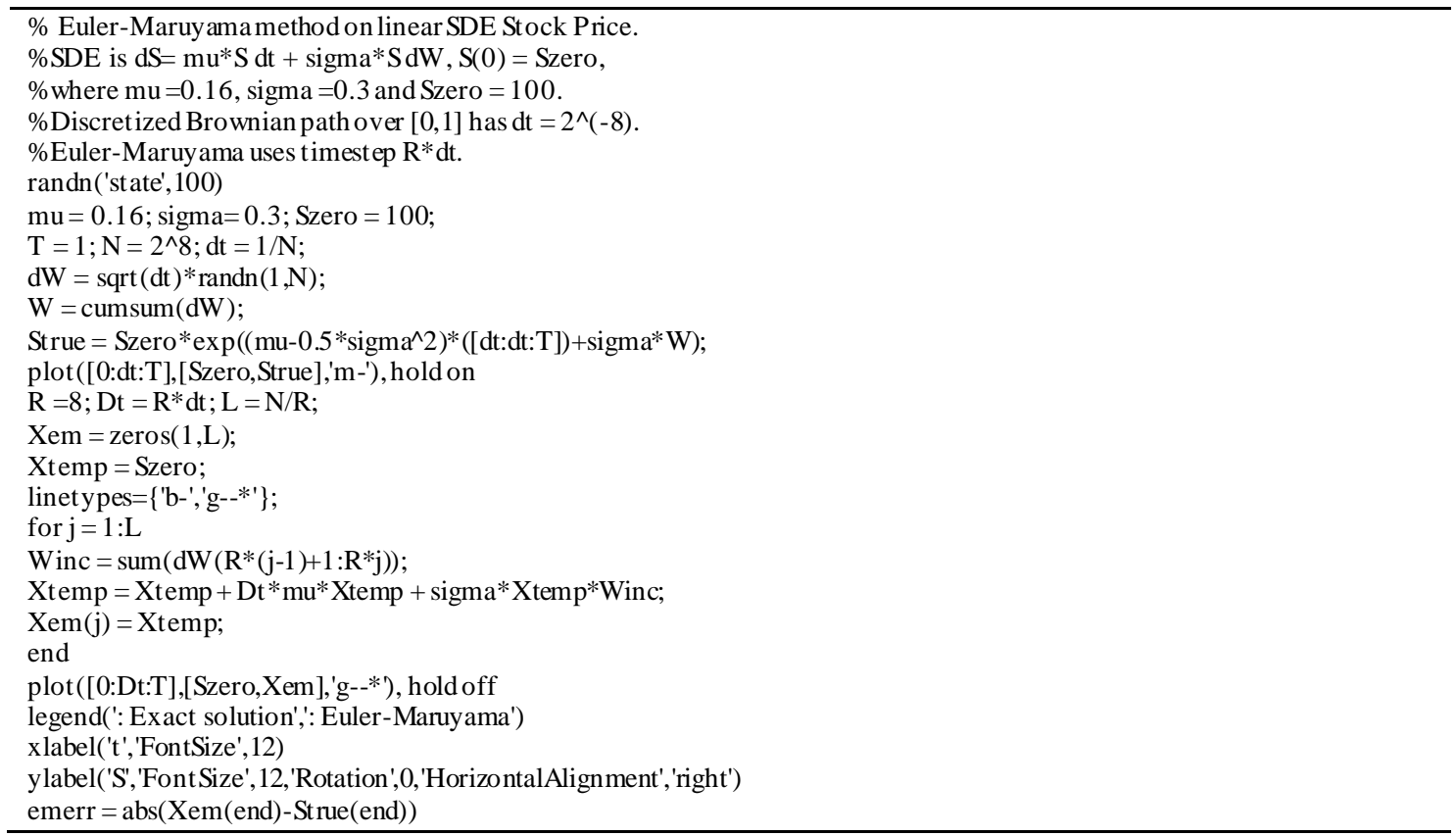

MATLAB 2. Exact solution and the solution based on Euler-Maruyama method on linear SDE stock price.

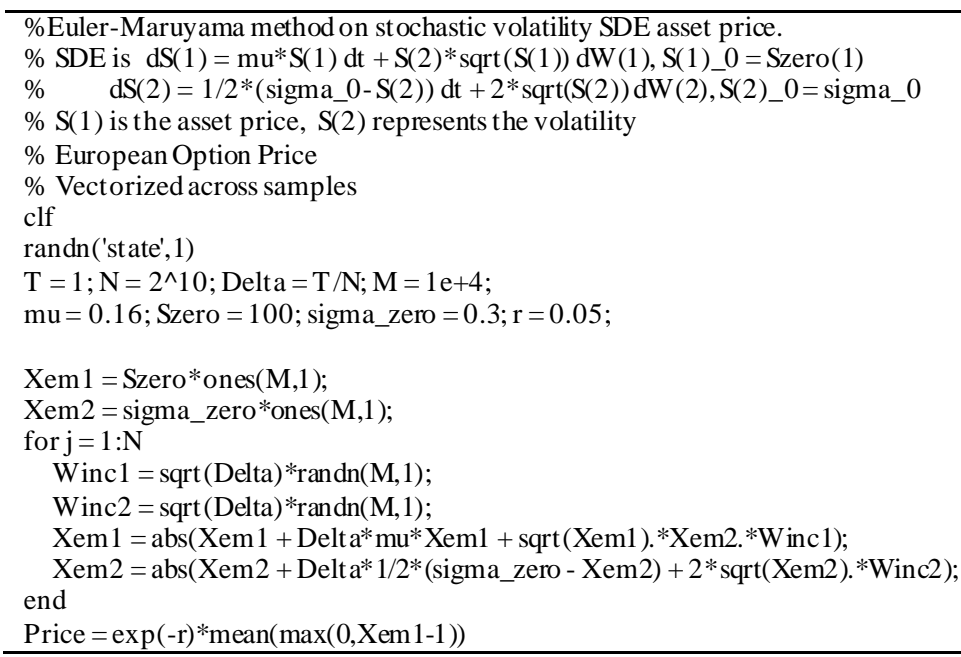

MATLAB 3. Estimating the value of European call option to buy the asset at the future time ${ }_{T}$ (using Euler-Maruyama method).

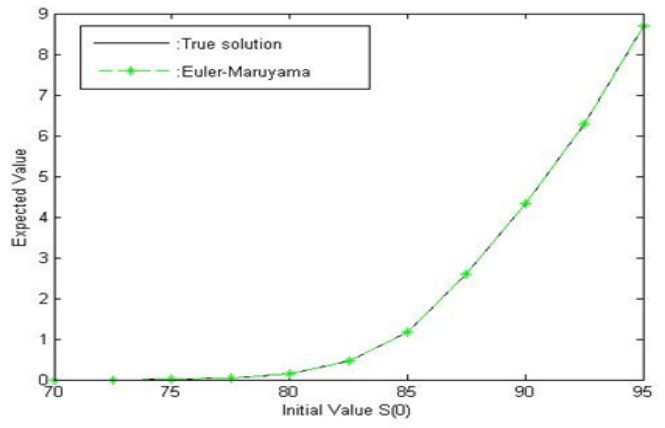

Fig.8. Euler-Maruy ama approximated payoff for a European call option.

Example 4. Look at the pair of following stochastic differential equations, the first equation is the asset price model, with as set variance $S_{t}^{2}$, following second equation:

$$
\left\{\begin{array}{l}
d S_{t}^{1}=\mu S_{t}^{1} d t+S_{t}^{2} \sqrt{S_{t}^{1}} d W_{t}^{1} \\
d S_{t}^{2}=\frac{1}{2}\left(\sigma_{0}-S_{t}^{2}\right) d t+2 \sqrt{S_{t}^{2}} d W_{t}^{2}
\end{array}\right.
$$

for $0 \leq t \leq 1$. The Brownian paths $W_{t}^{1}$ and $W_{t}^{2}$ are independent. We set $\mu=0.16, S_{0}^{1}=100$ and $S_{0}^{2}=\sigma_{0}=0.3$. We apply Euler-Maruyama method with step size $2^{-10}$ for $M=10,000$ paths. For estimating the value of European call option to buy the asset at the future time $T$, we form the average of $e^{-r} \max \left(0, S_{t=1}^{1}-1\right)$ overall paths with $r=0.05$. MATLAB 3 produce 110.6482 (value of European option to buy asset at future time ${ }_{T}$ ). So, the 
price of asset at $t=1$ exceeds $K=100$, the owner of the option may buy the as set at price $\mathrm{K}=100$ and immediately sell it at 110.6482 , hence gaining a profit of 10.6482 . If we approximate $e^{-r} E\left[\max \left(0, \bar{S}_{t}^{1}-1\right)\right]$ for equation (34), where $\bar{S}_{t}^{1}$ is the average of $S_{t}^{1}$ over $0 \leq t \leq 1$, this corresponds to pricing an Asian option. MATLAB 4 produce 102.2217 (value of Asian option to buy asset at future time ${ }_{T}$ ). If we exercise this option, we gain a profit of 2.2217 .

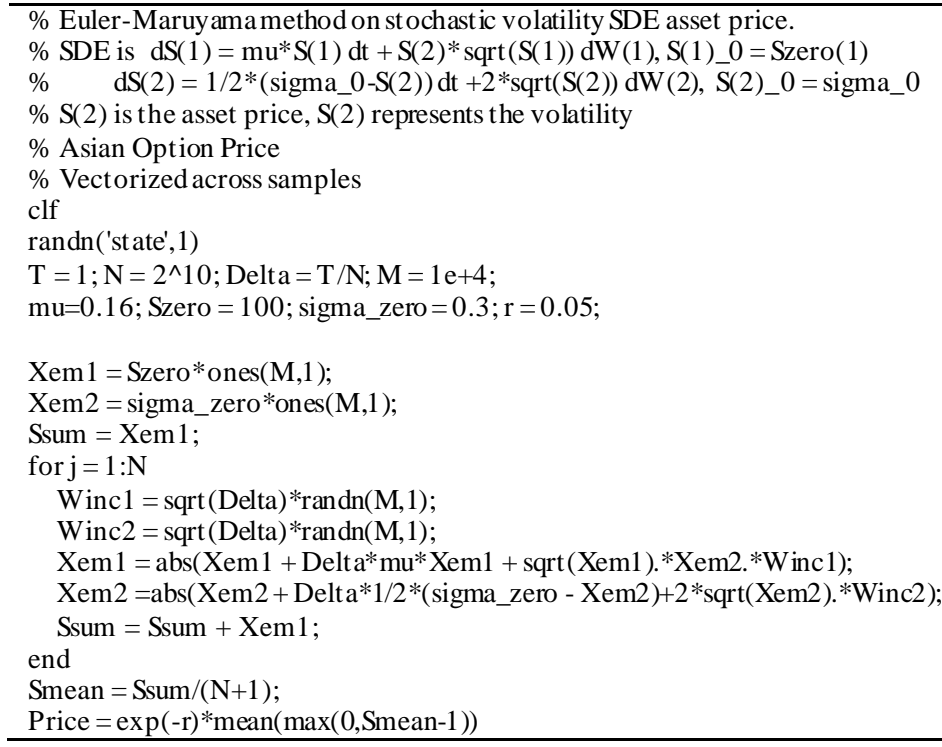

MATLAB 4. Estimating the value of Asian call option to buy the asset at the future time ${ }_{T}$ (using Euler-Maruyama method).

\section{CONCLUSION}

In this paper, we surveyed the applications of stochastic differential equations and Euler-Maruyama method for some financial problems. We found out the endpoint errors decreased with smaller delta $(\Delta t)$ for linear stochastic differential equation (the process for the stock price). Besides, we approximated square-root asset price model with different step sizes, because the solution to this equation is not explicit. Moreover, we estimated the value of European call option to buy the asset at the future time $T$, and we applied it for Asian option.

\section{REFERENCES}

[1] Desmond J. Higham. An Algorithmic Introduction to Numerical Simulation of Stochastic Differential Equations. SIAM Rev. Volume 43, Issue 3, 2001, PP. 525-546.

[2] Timothy. Sauer. Numerical Solution of Stochastic Differential equations in finance. To appear, Handbook of Computational Finance, 2008, Springer.

[3] Matthew, Rajotte. Stochastic Differential Equations and Numerical Application. Virginia Commonwealth University. Theses and Dissertations. 2014.

[4] Peter E. Kloeden, Ekhard. Platen. A survey of numerical methods for stochastic differential equation, Stochastic Hydrology and Hydraulics. 1989, Springer.

[5] Alain C. Nsengiy umva. Numerical Simulation of
Stochastic Differential Equations. Master's thesis, Lappeenranta University of Technology. 2013.

[6] Bernt. Oksendal. Stochastic Differential Equations, 6th ed, Springer-Verlag. 2013.

[7] Mark. Richardson. Stochastic Differential Equations Case Study, 2009.

[8] Bernt. Oksendal. Stochastic Differential Equations. An Introduction with Applications. Book. Fifth ed, Corrected Printing. 2000, Springer-Verlag Heidelberg New York.

[9] Gilsing. Hagen. Shardlow, Tony. SDELab: stochastic differential equations with MATLAB. Manchester nstitute for Mathematical Science School of Mathematics. The University of Manchester. 2006.

[10] Chaminda H. Baduraliya, Xuerong Ma. The EulerMaruy ama approximation for the asset price in the meanreverting-theta stochastic volatility model. 2012, ELSEVIER.

[11] Peter E. Kloeden, Ekhard. Numerical Solution of Stochastic Differential Equations. Book. 1995, Springer.

[12] Timothy. Sauer. Computational Solution of stochastic differential equations. Advanced Review, 2013, WIREs Comput Stat, 5:362-371.

[13] Sean. Fanning, Jay. parekh. Stochastic Processes and their Application to Mathematical Finance, 2004.

[14] Desmond J. Higham, Peter E. Kloeden.2002.M APLE and MATLAB for stochastic differential equations in finance.

[15] John C. Hull. options, futures and other derivatives. Book.7th ed, 2008.

[16] Picchini. Umberto. Simulation and Estimation of Stochastic Differential Equations with MATLAB. SDE TOOLBOX. User's Guide for version 1.4.1. 2007. 


\section{Authors' Profiles}

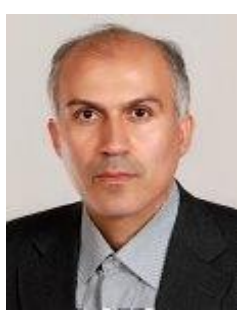

Dr. Hamid Reza Erfanian, Ph.D. in control and optimization from faculty of mathematical sciences, Ferdowsi University of Mashhad, Iran. $\mathrm{He}$ is professor at the faculty of mathematical sciences, University of Science and Culture, Tehran-Iran and his research interests are mainly on optimal control, optimization and financial mathematics.

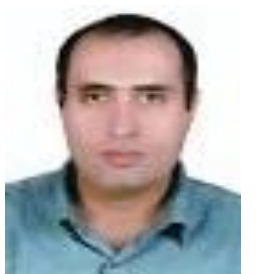

Dr. Mohammad Javad Abdi received his $\mathrm{PhD}$ and MSc in Applied Mathematics from University of Birmingham in England, and Sharif University of Technology in Iran, respectively. His research interests include Numerical Algorithms in Optimization. Portfolio Optimization Problems, and Cardinality and Rank Minimization Problems.

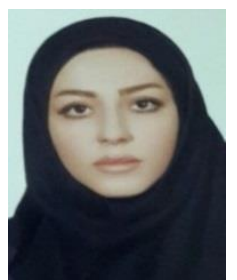

Mahshid Hajimohammadi was born in Iran in 1991. She received her bachelor degree in the field of pure mathematics at Alzahra University in 2013. She is a master student in the field of financial mathemathics at University of Science and Culture, Tehran-Iran.

How to cite this paper: Hamid Reza Erfanian, Mahshid Hajimohammadi, Mohammad Javad Abdi, "Using the EulerMaruyama Method for Finding a Solution to Stochastic Financial Problems", International Journal of Intelligent Systems and Applications (IJISA), Vol.8, No.6, pp.48-55, 2016. DOI: $10.5815 /$ ijisa.2016.06.06 